\title{
Downregulation of Orai1 expression in the airway alleviates murine allergic rhinitis
}

\author{
Yi Wang ${ }^{1}$, Lin Lin $^{2}$ and Chunquan Zheng ${ }^{1,3}$ \\ ${ }^{1}$ Department of Otorhinolaryngology-Head and Neck Surgery \\ Eye and ENT Hospital of Fudan University \\ Shanghai 200031, China \\ ${ }^{2}$ Department of Otorhinolaryngology-Head and Neck Surgery \\ Huashan Hospital of Fudan University \\ Shanghai 200040, China \\ ${ }^{3}$ Corresponding author: Tel, 86-21-64377134; \\ Fax, 86-21-64377151; E-mail, 96zheng @ sina.com \\ http://dx.doi.org/10.3858/emm.2012.44.3.013
}

Accepted 9 December 2011

Available Online 14 December 2011

Abbreviations: 2-APB, 2-aminoethoxydiphenyl borate; AR, allergic rhinitis; CRAC channels, $\mathrm{Ca}^{2+}$-release-activated $\mathrm{Ca}^{2+}$ channels; EAR3, eosinophil-associated, ribonuclease A family, member 3; ECP, eosinophil cation protein; ER, endoplasmic reticulum; LI, lenti-ORA/1 intervention; LPC, lysophosphatidylcholine; LTC4, leukotriene C4; LTC4S, leukotriene C4 synthase; $\mathrm{MOI}$, multiplicity of infection; NALT, nasal-associated lymphoid tissue; NECs, nasal epithelial cells; NLF, nasal lavage fluid; OVA-IgE, ovalbumin-specific IgE; RNAi, RNA interference; SOCE, store-operated $\mathrm{Ca}^{2+}$ entry; STIM1, stromal interaction molecule 1; TSLP, thymic stromal lymphopoietin

\begin{abstract}
Orai1 is the key subunit of the $\mathrm{Ca}^{2+}$-release-activated $\mathrm{Ca}^{2+}$ channel. Our previous report has demonstrated that Orai1 expression in the airway was upregulated in the ovalbumin (OVA)-induced allergic rhinitis (AR) mouse models. To observe whether inhibition of Orai1 expression in the airway could suppress symptoms in a murine model of $A R$ and to assess the impacts of this inhibition on the responses of local and systemic immunocytes, we administered recombinant lentivirus vectors that encoded shRNA against ORA/1 (lenti-ORA/1) into the nostrils of OVA-sensitized mice before the challenges, and analyzed its effect on allergic responses, as compared with the unsensitized mice and untreated AR mice. Administration of lenti-ORA/1 into the nasal cavity successfully infected cells in the epithelial layer of the nasal mucosa, and significantly de-
\end{abstract}

creased the frequencies of sneezing and nasal rubbing of the mice. Protein levels of leukotriene C4, OVA-specific $\lg E$, and IL-4 in the nasal lavage fluid and serum and eosinophil cation protein in the serum were also significantly reduced by lenti-ORA/1, as were the mRNA levels of these factors in the nasal mucosa and spleen. These data suggested that administration of lenti-ORA/1 into the nasal cavity effectively decreased Orai1 expression in the nasal mucosa, alleviated AR symptoms, and partially inhibited the hyperresponsiveness of the local and systemic immune cells including T cells, B cells, mast cells and eosinophils that are involved in the pathogenesis of AR.

Keywords: calcium channels; disease models, animal; lentivirus; mice; ORAl1 protein, human; rhinitis; RNA interference

\section{Introduction}

Allergic rhinitis (AR) is allergic inflammation in the upper airway associated with the hyperresponsiveness of several types of immune cells. With the help of antigen presenting cells (APCs), an allergen triggers a Th2-predominant immune response that leads to the generation of memory $B$ cells that produce antigen-specific IgE. This IgE binds to mast cells, and subsequent exposure to the allergen provokes these lgE-binding mast cells to release inflammatory mediators, such as histamine and leukotriene C4 (LTC4), that induce allergic reactions. Eosinophilic infiltration also plays a role in persistent airway inflammation and remodeling. Mainstream therapy for AR consists of disease control through the use of anti-inflammatory agents, glucocorticoidbased drugs, antihistamines, and antileukotrienes. Although these drugs relieve symptoms in most patients, they do not cure the underlying disease. Therefore, the challenge to develop safe, new therapeutic agents that target the root of the pathogenesis of AR remains.

There is growing evidences that in cells of the immune system, $\mathrm{Ca}^{2+}$ entry from the extracellular medium is induced by the depletion of endoplasmic reticulum (ER) $\mathrm{Ca}^{2+}$ stores and mediated by speci- 
alized store-operated $\mathrm{Ca}^{2+}$ channels called $\mathrm{Ca}^{2+}$-release-activated $\mathrm{Ca}^{2+}$ (CRAC) channels (Hoth and Penner, 1992). The molecular composition of these channels and the mechanisms linking store depletion to channel activation remained obscure until 2005 when stromal interaction molecule 1 (STIM1) was identified as the mammalian $\mathrm{ER} \mathrm{Ca}{ }^{2+}$ sensor (Liou et al., 2005). Orai1 was later identified as a key subunit of the CRAC channel pore (Prakriya et al., 2006). STIM1 is a single pass ER-resident protein with a $\mathrm{Ca}^{2+}$ binding EF hand domain that faces the ER lumen. $\mathrm{Ca}^{2+}$ dissociates from the EF hand during store depletion, which leads to STIM1 oligomerization. The oligomers then migrate and form punctate-like structures below the plasma membrane. The oligomers bind to Orai1, which is diffusely distributed in the plasma membrane at rest, and activate it, causing formation of the channel pore. Mammalian cells express two other closely related homologues of Orai1, Orai2 and Orai3, which appear to function similarly in mediating store-operated $\mathrm{Ca}^{2+}$ entry (SOCE) when co-expressed with STIM1, but their $\mathrm{Ca}^{2+}$ currents are 2to 3-fold weaker than the $\mathrm{Ca}^{2+}$ current mediated by Orai1 (Mercer et al., 2006). The discovery of these proteins has led to rapid progress in the illumination of the molecular mechanisms of the aforementioned pathway and its functions in various tissues.

The process of store-operated $\mathrm{Ca}^{2+}$ influx is essential for both the short- and long-term responses of immune system cells. Short-term responses include the degranulation of mast cells and the killing of target cells by effector cytolytic T cells, whereas long-term responses typically involve changes in gene transcription through the $\mathrm{Ca}^{2+}$ /calcineurin/nuclear factor of activated T cells signaling pathway and include T- and B-cell proliferation and differentiation (Parekh and Putney, 2005; Gwack et al., 2007). These responses are considered crucial processes in the induction and development of AR.

In our first study, it was revealed that Orai1 was expressed in murine nasal mucosa, nasal-associated lymphoid tissue (NALT), trachea mucosa, and spleen and that Orai1 expression was upregulated in AR mice compared to controls (Lin et al., 2010), suggesting that the SOCE pathway may contribute to the pathogenic mechanisms of AR and could be a potential therapeutic target for the treatment of AR. In our subsequent study, we demonstrated that the administration of 2-aminoethoxydiphenyl borate (2-APB), which inhibits the activation of CRAC, into the nostrils of AR mice could alleviate allergic symptoms and reduce the number of eosinophils that infiltrate into the nasal mucosa. Furthermore, the administration suppressed
Orai1 expression in nasal mucosa and decreased allergic inflammatory mediators in the nasal lavage fluid (NLF) (Lin et al., 2011). However, 2-APB has been known to interfere with a variety of transport processes besides CRAC channels, including $\mathrm{K}^{+}$ channels (Wang et al., 2002) and mitochondrial $\mathrm{Ca}^{2+}$ efflux (Prakriya and Lewis, 2001). This lack of specificity could lead to questions about the validity of our results obtained with 2-APB. Furthermore, the temporary inhibition of CRAC channels by 2-APB may lead to only transient inhibition of $A R$ symptoms.

Based on our previous findings, the present study was designed to observe whether inhibition of Orai1 expression specifically in the airway could suppress allergic symptoms in murine models of $A R$ and how this reduced expression impacts the responses of local and systemic immune cells including T cells, B cells, mast cells and eosinophils. We hope that the findings presented here may provide further insight on the role the SOCE pathway plays in the pathogenesis of AR and render some clues to the development of novel therapies.

\section{Results}

\section{Transfection efficacies and Orai1 transcription in- hibition efficacies of Lenti-Orai1 and Lenti-GFP}

The transfection efficacies of recombinant lentivirus vectors that encoded shRNA against ORAl1 as well as green fluorescent protein (GFP) (lenti-ORAl1) and lentivirus vectors only encoded GFP (lenti-GFP) were analyzed by fluorescence-activated cell sorting (FACS) for GFP expressed in the transfected PANC cells. When the multiplicity of infection (MOI) is 10 , the transfection efficacy of Lenti-ORAl1 is $98.28 \%$, and the transfection efficacy of Lenti-GFP is $98.75 \%$. Orai 1 transcription inhibition efficacies of Lenti-ORAl1 and Lenti-GFP were determined by measuring the Orai1 mRNA levels of control PANC cells, PANC cells transfected by lenti-ORAl1 and lenti-GFP, respectively. When the $\mathrm{MOI}$ is 10 , the inhibition efficacy of Lenti-ORAl1 is $90.5 \%$, and that of lenti-GFP is $-1.4 \%$ (Figure $1 \mathrm{~A}$ ).

\section{The kinetics of the inhibition efficacy of Orai1 transcription by in vivo transfection with Lenti-Orai1}

The in vivo inhibition efficacies of Lenti-ORAl1 varied according to the duration of the transfetion. The kinetics of the inhibition efficacy were showed in Figure 1B. Since the inhibition efficacy reached a maximum in the third day after the transfection, which is $58.5 \%$ (Figure 1B), we choosed the timing 

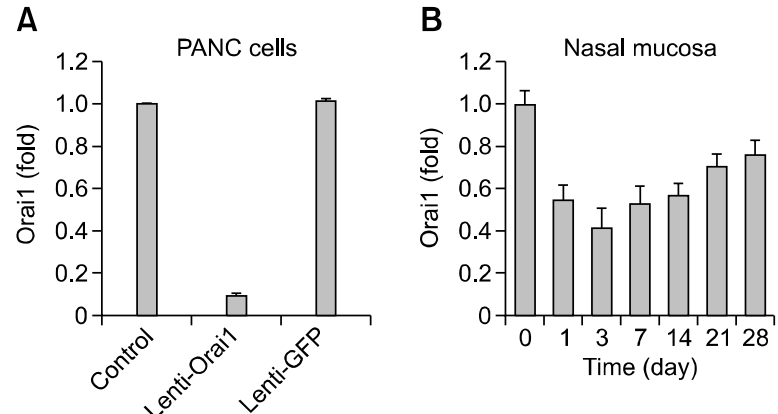

Figure 1. Orai1 transcription inhibition efficacies. Orai1 transcription inhibition efficacies of lenti-Orai1 and lenti-GFP in PANC cells (A), and the kinetics of the inhibition efficacy of Orai1 transcription of lenti-Orai1 in the mouse nasal mucosa (B). Orai1 transcription level of each sample was assessed by real-time RT-PCR. Each value represents mean \pm SEM.

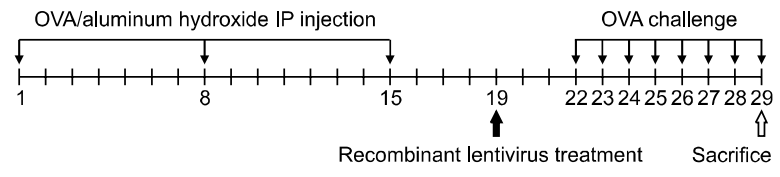

Figure 2. Experimental design. The mice were randomly divided into five groups: normal control , normal mice with Lenti-Orai1 interferenced (NLI), mice with allergic rhinitis (AR), AR mice with lenti-GFP interferenced (AR-GFP), and AR mice with lenti-Orai1 interferenced (AR-LI). The mice of AR, AR-GFP and AR-LI groups received ovalbumin (OVA)/aluminum hydroxide introperitoneal injection as sensitization on day 1, 8 and 15 , followed with OVA intronasal challenges on day 22-29. The mice in group $N L I$ and AR-LI were given the single dose of lenti-Orai1 on day 19; meanwhile, mice in group AR-GFP were given a single dose of lenti-GFP.
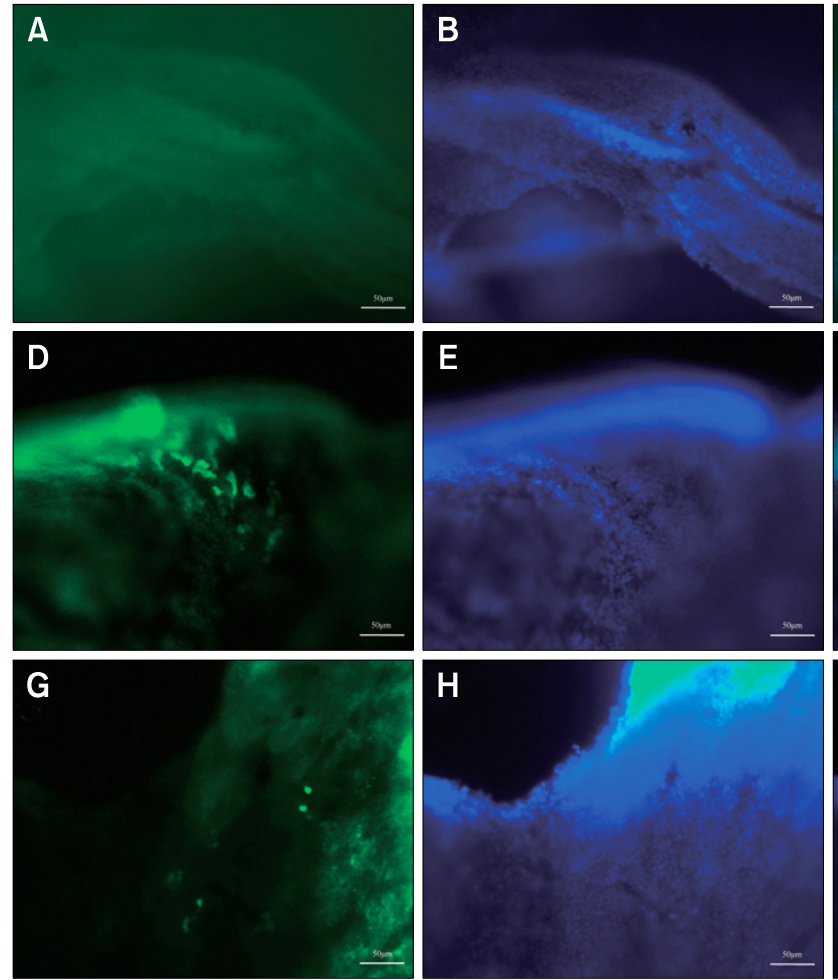
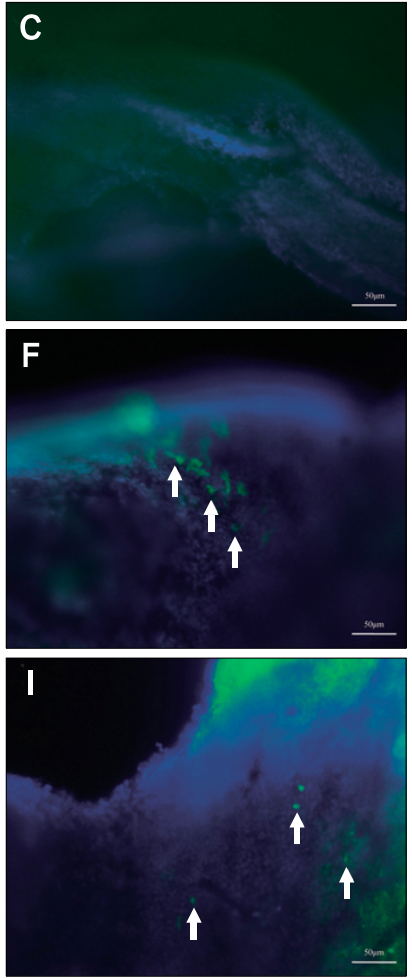

Figure 3. GFP expression in the nasal mucosa. The arrowheads indicate GFP expression in transfected cells in the epithelial layer of the nasal mucosa of mice intranasally administered lenti-Orai1 $(F)$ or lenti-GFP (I). There was no GFP expression in the nasal mucosa of the mice in the normal control group and AR group (C). A, D, G: GFP. B, F, H: DAPI. C, F, l: Merge. Scale bar $=50 \mu \mathrm{m}$ for transfecting the mice in NLI, AR-GFP and AR-LI groups with corresponding lentiviruses three days before accepting the first challenge with ovalbumin.

\section{Administration of recombinant lentivirus to the nasal cavity via inhalation-driven instillation successfully transfected cells in the nasal mucosa}

The experimental design is summerized in Figure 2. To determine if the recombinant lentivirus was successfully transfected into cells in the airway, three mice of each group were sacrificed on the first day of the challenge, their nasal mucosa and spleens were carefully dissected and observed under fluorescence microscope to determine if the green fluorescence of GFP was expressed in potentially transfected cells. The inhalation-driven instillation of lentiviruses established GFP transgene expression in the nasal mucosas of NLI, AR-GFP and AR-LI mice, while no green fluorescence was observed in either the normal control mice or the AR mice. Similar to the findings of Stocker et al (Stocker et al., 2009), the cells expressing GFP were mainly dispersed within the anterior regions 
of the epithelial layer in the treated nasal airways (Figure 3). The spleen of each mouse was also examined, and no green fluorescence signal was observed in any spleen, including the spleens of the $\mathrm{NLI}, \mathrm{AR}-\mathrm{GFP}$ and AR-LI groups. This implies that

A

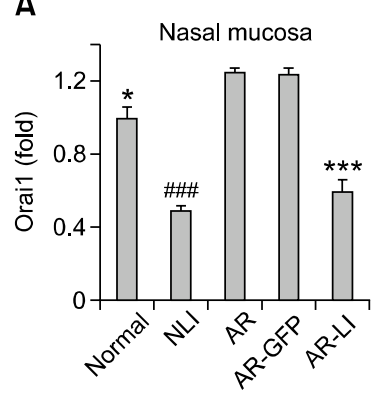

B

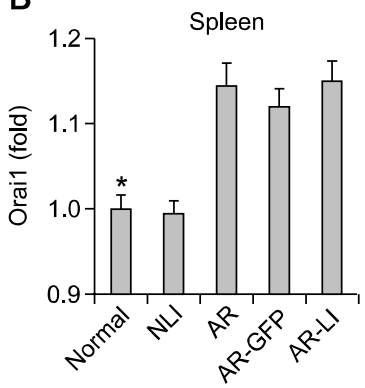

C

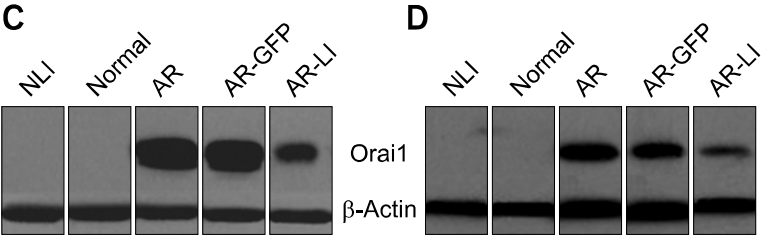

Figure 4. The mRNA and protein expression of Orai1. Orai1 transcript levels in the nasal mucosa (A) and spleen (B) of mice in each group were assessed by real-time RT-PCR. Each value represents mean \pm SEM. ${ }^{*} P<0.05$ vs. AR group; ${ }^{* * *} P<0.001$ vs. AR group; ${ }^{\# \#} P<0.001$ vs. normal control group. Orai1 expression levels in the nasal mucosa (C) and spleen (D) of the mice were determined by Western blotting. administration of the lentivirus vector through the nostrils did not lead to the transfection of splenocytes.

\section{Administration of lenti-ORA/1 siRNA downregulated Orai1 expression in the nasal mucosa and spleen}

The extracted Orai1 mRNA from the nasal mucosa and spleens of the mice were evaluated by real-time RT-PCR. Orai1 mRNA levels in the nasal mucosa and spleen were upregulated in AR mice compared to normal controls $(P<0.05$, and $P<$ 0.05 , respectively; Figures $4 \mathrm{~A}$ and $4 \mathrm{~B}$ ). In addition, lenti-ORAl1 treatment significantly reduced the levels of Orai1 mRNA in the nasal mucosa of AR-LI mice compared to the AR group $(P<0.001$, AR-LI vs. AR; Figure 4A), as well as the levels in the NLI mice compared to the normal ones $(P<0.001$, $\mathrm{NLI}$ vs. normal; Figure $4 \mathrm{~A})$. The treatment of the sensitized and challenged mice with lenti-GFP didn't change their Orai1 mRNA levels in the nasal mucosa compared to AR mice $(P>0.05$, Figure $4 A)$. Both administration of lenti-ORAl1 and lenti-GFP to the nasal cavity had no effect on Orai1 mRNA levels in the spleen $(P>0.05, P>0.05, P$ $>0.05$, NLI vs. normal, AR-GFP vs. AR, AR-LI vs. $A R$, respectively; Figure $4 B$ ), which was consistent with our observation that the splenocytes had not been transfected by the lentiviruses.

The proteins extracted from the nasal mucosa and spleens of the mice were evaluated by Western
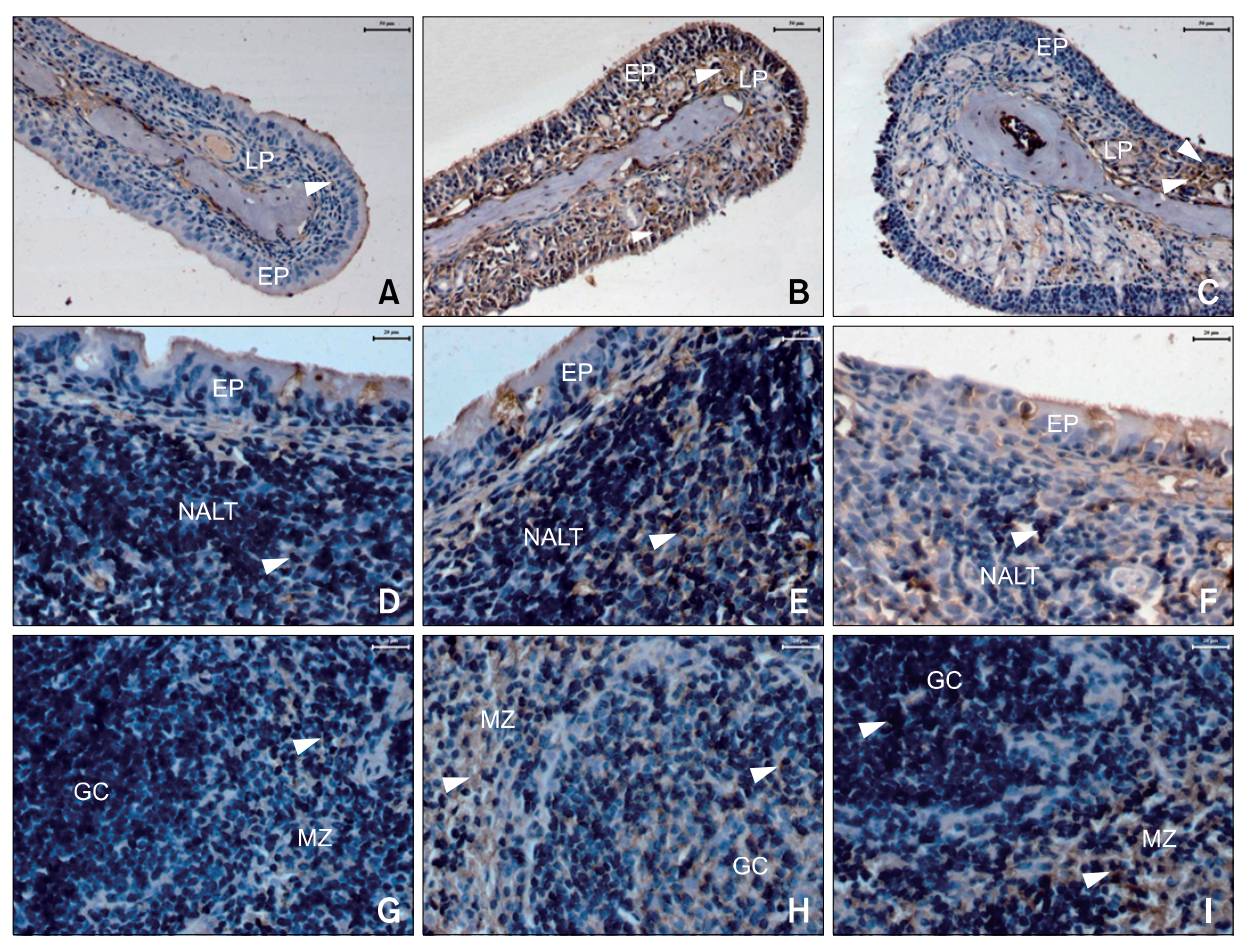

Figure 5. Orai1 distribution. Arrowheads indicate immunoreactivity for Orai1 in cells in normal controls $(A, D, G)$, allergic rhinitis $(A R)$ mice $(B, E, H)$, and lentivirus intervention (AR-LI) mice $(C, F, I)$ in the nasal mucosa (A-C), NALT $(D-F)$, and spleen (G-I). EP, epithelial layer of nasal mucosa; LP, lamina propria of the nasal mucosa; $\mathrm{GC}$, germinal center; $M Z$, marginal zone. Scale bar $=50 \mu \mathrm{m}$ for $\mathrm{A}-\mathrm{C}$, $20 \mu \mathrm{m}$ for D-I. 
Table 2. Total cell number and diffrrential cell numbers in NLF of the mice

\begin{tabular}{lcrrrr}
\hline \multicolumn{1}{c}{ Group } & Normal & \multicolumn{1}{c}{ NLI } & \multicolumn{1}{c}{ AR } & \multicolumn{1}{c}{ AR-GFP } & AR-LI \\
\hline Epithelial cells $\left(\times 10^{4} / \mathrm{ml}\right)$ & $0.77 \pm 0.26^{*}$ & $0.55 \pm 0.20$ & $6.89 \pm 1.92$ & $7.02 \pm 2.00$ & $1.30 \pm 0.54^{*}$ \\
Lymphocytes $\left(\times 10^{4} / \mathrm{ml}\right)$ & $2.29 \pm 0.55^{*}$ & $1.99 \pm 0.47$ & $10.37 \pm 1.99$ & $10.89 \pm 2.97$ & $3.35 \pm 1.08^{*}$ \\
Eosinophils $\left(\times 10^{3} / \mathrm{ml}\right)$ & $0.36 \pm 0.11^{*}$ & $0.31 \pm 0.15$ & $9.18 \pm 2.27$ & $9.14 \pm 2.41$ & $1.22 \pm 0.57^{*}$ \\
Neutrophils $\left(\times 10^{4} / \mathrm{ml}\right)$ & $0.51 \pm 0.13^{*}$ & $0.44 \pm 0.14$ & $4.41 \pm 1.39$ & $3.42 \pm 0.59$ & $1.22 \pm 0.49^{*}$ \\
Total cells $\left(\times 10^{4} / \mathrm{ml}\right)$ & $3.60 \pm 0.89^{*}$ & $3.00 \pm 0.82$ & $22.60 \pm 4.83$ & $22.25 \pm 5.50$ & $6.00 \pm 2.00^{*}$ \\
\hline
\end{tabular}

This table represents the mean and the standard deviation of determinations.

${ }^{*}$ Statistical significance was noted as compared with AR mice, $P<0.05$.

Table 1. Immunoreactivity to Orai1 in the nasal mucosa, NALT, and spleen

\begin{tabular}{|c|c|c|c|c|}
\hline \multirow{2}{*}{ No. } & \multicolumn{2}{|c|}{ Nasal mucosa } & \multirow{2}{*}{$\frac{\text { NALT }}{\text { IC }}$} & \multirow{2}{*}{$\begin{array}{c}\text { Spleen } \\
\text { IC }\end{array}$} \\
\hline & $\mathrm{EC}$ & LC & & \\
\hline \multicolumn{5}{|c|}{ Normal controls } \\
\hline 1 & + & + & $+/-$ & + \\
\hline 2 & - & - & - & + \\
\hline 3 & - & - & - & + \\
\hline 4 & + & + & $+/-$ & + \\
\hline 5 & - & - & - & + \\
\hline 6 & - & - & - & + \\
\hline \multicolumn{5}{|l|}{ AR group } \\
\hline 7 & +++ & +++ & ++ & ++ \\
\hline 8 & +++ & +++ & ++ & ++ \\
\hline 9 & +++ & +++ & ++ & +++ \\
\hline 10 & +++ & +++ & ++ & ++ \\
\hline 11 & +++ & +++ & ++ & +++ \\
\hline 12 & +++ & +++ & ++ & ++ \\
\hline \multicolumn{5}{|l|}{ LI group } \\
\hline 13 & - & + & + & + \\
\hline 14 & + & ++ & ++ & ++ \\
\hline 15 & - & + & + & + \\
\hline 16 & - & + & + & + \\
\hline 17 & - & + & + & ++ \\
\hline 18 & + & ++ & + & ++ \\
\hline
\end{tabular}

The numbers 1-18 each represent an individual mouse; EC, cells in the epithelial layer of the nasal mucosa; LC, cells in the lamina propria of the nasal mucosa; IC, immune cells; -, the absence of positivity; +, weakly positive; ++, definitely positive; +++, strongly positive.

Blotting. The results from the nasal mucosa showed a much higher level of Orai1 protein expression in the AR group compared to the normal group and an apparently decreased level of Orai1 in the AR-LI group compared to the AR group (Figure $4 C)$. Both of the expression of the normal mice and NLI mice are too low to detect. The Orai1 expression level of the mice in GFP group was similar to that of the AR mice. The results in the spleen were similar to that in the nasal mucosa (Figure 4D).
A
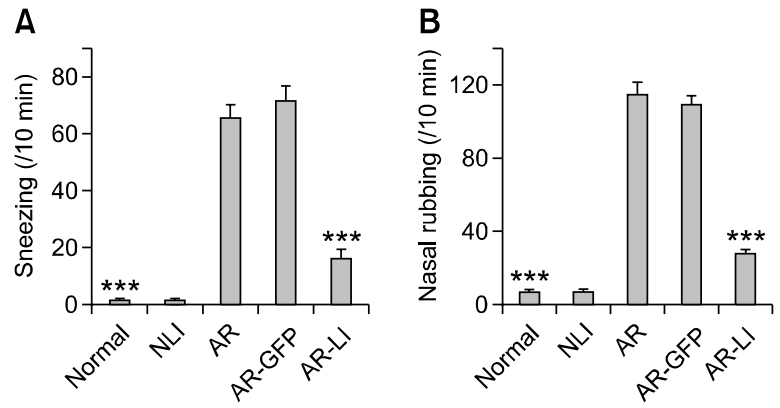

Figure 6. Nasal allergic symptoms. The number of sneezes $(A)$ and nasal rubbing episodes (B) were determined for 10 min immediately following the final challenge for the mice. Each value represents the mean \pm SEM of each group. ${ }^{*} P<0.05 ;{ }^{* * *} P<0.001$ vs. the AR group.

\section{The distribution of Orai1 changed after lenti-ORAl1 siRNA administration}

Immunohistochemical staining of Orai1 was performed to demonstrate its distribution in murine nasal mucosa, NALT, and spleen. Orai1 was localized primarily in the plasma membrane of cells in these tissues. In the nasal mucosa of AR mice, Orai1 was located in cells in the epithelial layer and the ductal epitheliums of the submucosal glands, while it was barely detectable in normal mice (Figures $5 \mathrm{~A}$ and $5 B$ ). In AR-LI mice, compared to the AR group, a marked reduction of Orai1 was observed in cells in the epithelial layer of the nasal mucosa and a less robust, but still definitive reduction was observed in cells in the lamina propria (Figure $5 \mathrm{C}$ ). Furthermore, Orai1 protein was localized in the plasma membrane of immunocytes in the NALT and spleen (Figures $5 \mathrm{E}$ and $5 \mathrm{H}$ ), and its immunostaining was more intense in the AR group than the AR-LI group (Figures $5 \mathrm{~F}$ and $5 \mathrm{I}$ ). The immunostaining was hard to detect in the NALT of the normal control group, and was only weakly positive in the marginal zones of the spleens of normal group (Figures 5D and 5G). On the other hand, although the Orai1 expression in the lymphocytes residing in the marginal zones in the spleens of AR-LI mice was still distinctly positive, its expression in the cells residing in the germinal cen- 
ters of these mice was much lower than that in the AR mice (Figure 5I). The detailed distribution of Orai1 immunopositive cells is presented in Table 1.

\section{Downregulation of Orai1 expression in the airway alleviated symptoms of allergic rhinitis in mice}

To examine whether the downregulation of Orai1 expression in the airway in vivo had protective effects on allergic rhinitis, the number of sneezes was recorded after the last OVA challenge. The AR group showed an apparent increase in the number of sneezes ( $65.60 \pm 4.88$ counts $/ 10 \mathrm{~min}) \mathrm{com}$ pared to the normal control group that had only received one dose of challenge on day 29 (1.70 \pm 0.42 counts $/ 10 \mathrm{~min}$ ). The average sneeze count of AR-LI mice was $16.20 \pm 3.28$ counts $/ 10 \mathrm{~min}$, and this was significantly less than that of AR mice $(P$ $<0.001$; Figure $6 \mathrm{~A}$ ). At the same time, the number of nasal rubbings was recorded by another observer. Similarly, AR mice showed an obvious increase in the number of nasal rubbings (115.00 \pm 6.24 counts $/ 10 \mathrm{~min}$ ) compared to the normal mice (7.30 \pm 1.25 counts/10 min), and AR-LI mice had significantly less nasal rubbings $(27.90 \pm 2.12$ counts $/ 10 \mathrm{~min})$ than $\mathrm{AR}$ mice $(P<0.001$; Figure $6 \mathrm{~B})$. However, the administration of lenti-GFP could neither decrease the number of sneezing nor the number of nasal rubbings. These results clearly indicated that the downregulation of Orai1 expression in the airway by a single intranasal administration of lenti-ORAl1 alleviated allergic symptoms in mice.

\section{Total and differential inflammatory cell numbers in NLF}

Total and differential cell number were counted in NLF obtained from each mice (Table 2). The total cell number in the NLF of AR mice was significantly greater than that of normal mice. The numbers of epithelial cells, lymphocytes, eosinophils and neutrophils in NLF were all significantly greater in AR mice than in normal mice. Most predominant difference was observed in the number of eosinophils. The total and differential cell numbers, especially the number of eosinophils in the NLF of AR-LI mice were all decreased compared to AR mice.

\section{Levels of allergic inflammatory cytokines and im- munoglobulins in the NLF and serum decreased after treatment with lenti-ORA/1}

We examined LTC4, eosinophil cation protein (ECP), ovalbumin-specific IgE (OVA-lgE), and IL-4
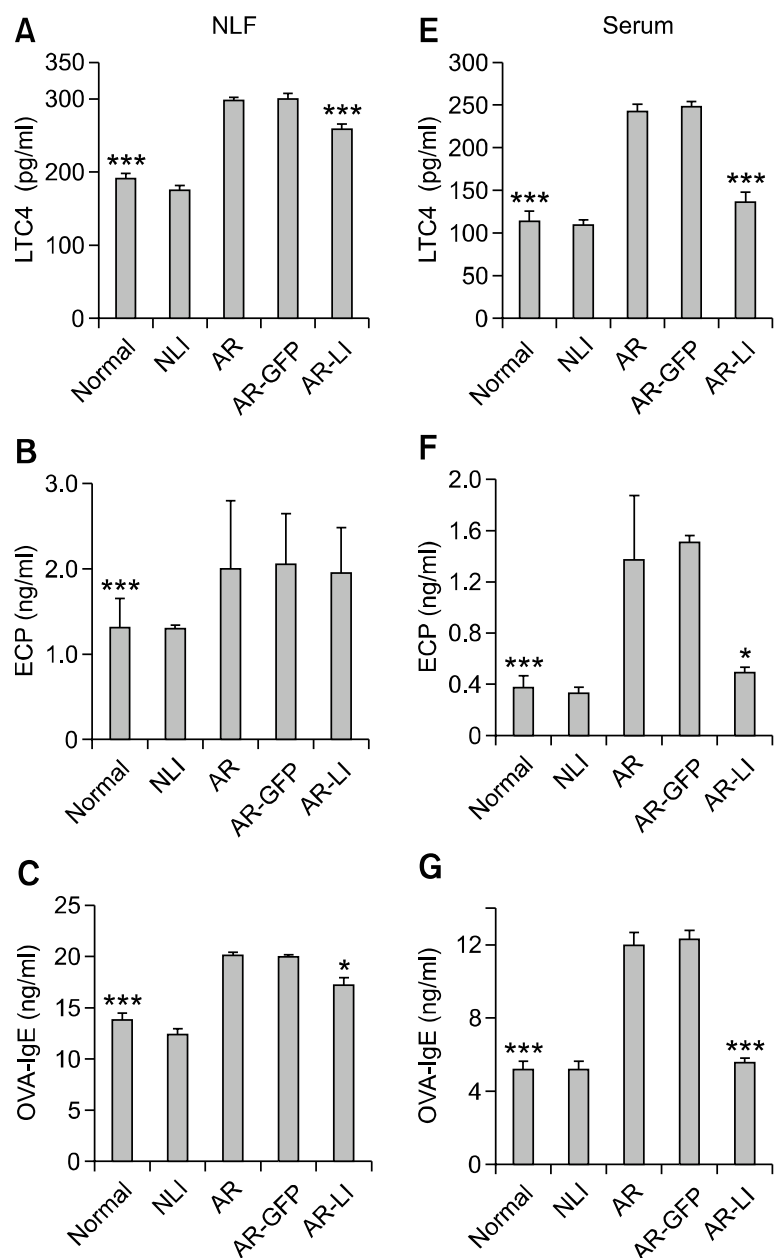

$\mathbf{F}$

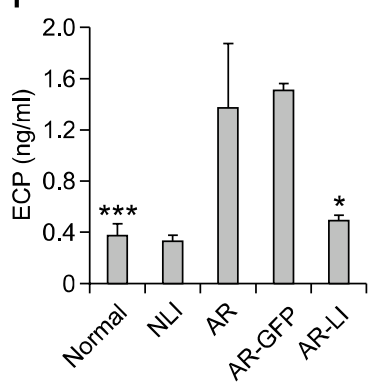

G

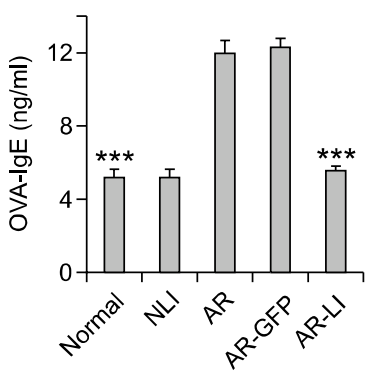

D

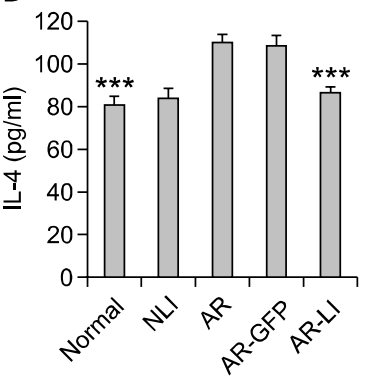

$\mathrm{H}$

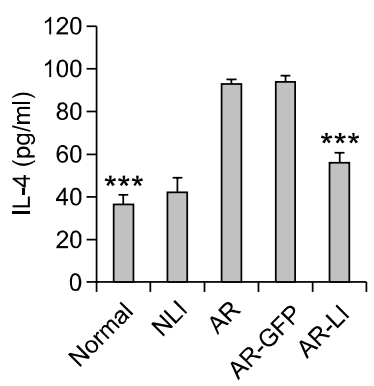

Figure 7. ELISA analysis of LTC4, ECP, OVA-lgE and IL-4 in the NLF ( $A-D$, respectively) and serum (E-H, respectively) of the mice. Each value represents the mean \pm SEM of each group. ${ }^{*} P<0.05$; ${ }^{* * *} P<0.001$ vs. the AR group.

levels, which represent the function of mast cells, eosinophils, B cells and Th2 cells respectively, in the NLF and serum of the mice. The NLF and serum levels of LTC4, ECP, OVA-lgE, and IL-4 of AR mice were all elevated compared to those of the normal group (all $P<0.001$; Figure 7). The NLF levels of LTC4, OVA-IgE, and IL-4 of the AR-LI 

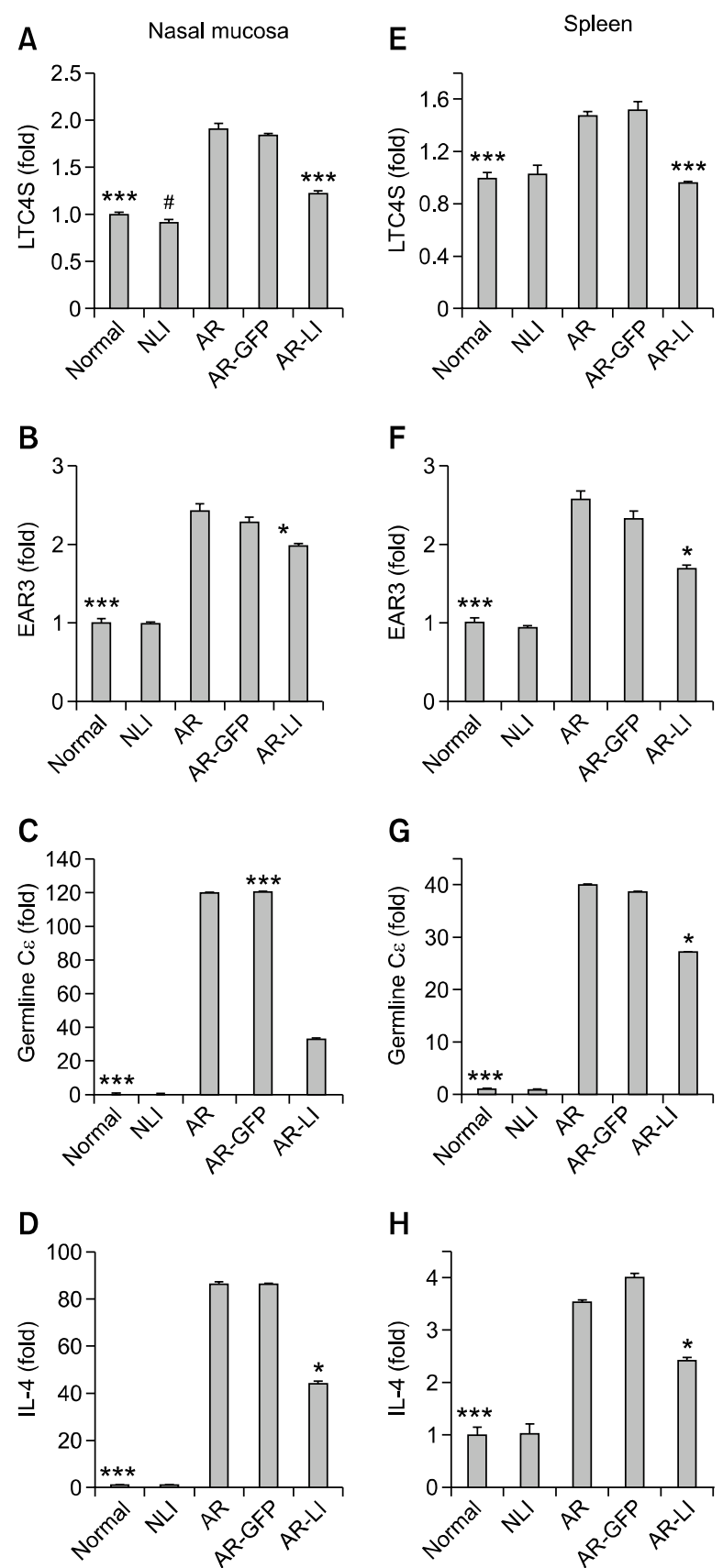

$\mathrm{H}$

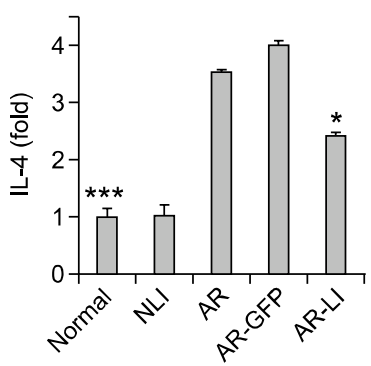

Figure 8. Real-time RT-PCR analysis of mRNA levels of LTC4S, EAR3, germline $C \varepsilon$, and $\mathrm{IL}-4$ in the nasal mucosa (A-D, respectively) and spleen (E-H, respectively) of the mice. Each value represents the mean \pm SEM of each group. ${ }^{*} P<0.05 ;{ }^{* * *} P<0.001$ vs. the AR group; ${ }^{\#} P$

$<0.05$ vs. normal control group.

group were significantly reduced compared to those of AR mice $(P<0.001, P<0.05$, and $P<$ 0.001 , respectively; Figures $7 \mathrm{~A}, 7 \mathrm{C}$ and $7 \mathrm{D}$ ). However, there was not a significant difference between the AR-LI and AR groups in the NLF level of ECP $(P>0.05$; Figure 7B). The serum levels of

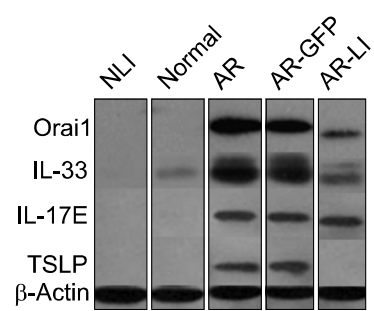

Figure 9. Protein expression of IL-33, IL-17E and TSLP. Expression levels of IL-33, IL-17E and TSLP in the nasal mucosa of the mice in each group were determined by Western blotting.

LTC, ECP, OVA-IgE, and IL-4 in AR-LI mice were all significantly reduced compared to those in the AR group $(P<0.001, P<0.05, P<0.001$, and $P$ $<0.001$, respectively; Figure $7 \mathrm{E}-7 \mathrm{H}$ ). These results showed that suppression of Orai1 expression in the nasal mucosa inhibited both local and systemic allergic responses.

Lenti-ORA/1 inhibited the induction of leukotriene C4 synthase (LTC4S), eosinophil-associated, ribonuclease A family, member 3 (EAR3), IL-4, and Germline $C_{\varepsilon}$ transcripts in the nasal mucosa and spleen in AR-LI mice

To investigate the effect of the intranasal administration of lenti-ORAl1 on mRNA levels of LTC4S, EAR3, the germline C transcript, and IL-4 in the nasal mucosa and in spleen, we measured levels of these transcripts by real-time RT-PCR. In the AR mice, compared to normal controls, there were increases in all four transcripts in both the nasal mucosa and spleen (all $P<0.001$; Figure 8). The administration of lenti-ORAl1 resulted in a reduction of these transcripts in the nasal mucosas (LTC4S, $P<0.001$; EAR3, $P<0.05$; germline $C$ transcript, $P<0.001 ;$ IL-4, $P<0.05$; all vs. the AR group; Figures 8A-8D) and the spleens (LTC4S, $P<$ 0.001 ; EAR3, $P<0.05$; germline $C$ transcript, $P<$ 0.05 ; IL-4, $P<0.05$; all vs. the AR group; Figures $8 \mathrm{E}-8 \mathrm{H})$ in $\mathrm{AR}-\mathrm{LI}$ mice compared to AR mice, but have no influence on NLI mice compared to normal mice. These findings suggested that RNA interference of Orai1 in the nasal mucosa limited allergic responses in the nasal mucosa and spleen.

\section{Downregulation of Orai1 expression reduced the ex- pression of IL-33 and thymic stromal lymphopoietin (TSLP) in AR-LI mice}

The expressions of IL-33, IL-17E and TSLP in the nasal mucosa of the mice were evaluated by Western Blotting. The results showed higher levels of IL-33, TSLP and IL-17E expression in the AR 
group compared to the normal group and slightly decreased levels of IL-33 and TSLP in the AR-LI group compared to the AR group (Figure 9). However, the level of IL-17E in AR-LI mice was not influenced by the administration of lenti-ORAl1 compared to AR mice. The IL-33, IL-17E, and TSLP expression levels of the AR-GFP mice were similar to that of the AR mice.

\section{Discussion}

$\mathrm{Ca}^{2+}$ signals are vital in the function of many cell types of the immune system, including $T$ cells, B cells, NK cells, mast cells, dendritic cells, and macrophages, and they control cell functions as diverse as differentiation, proliferation, gene expression, cell motility, and the secretion of vesicles that contain cytokines, cytotoxic proteins, and/or proinflammatory proteins (Feske, 2007; Gwack et al., 2007). In immune cells, $\mathrm{Ca}^{2+}$ signaling results from the engagement of antigen receptors at the cell surface. Therefore, they directly determine the intensity of the immune response triggered by an antigen (Feske, 2009). These responses are considered crucial processes in the induction and development of AR.

We administered 2-APB into the nostrils of AR mice to partially block the activation of CRAC channels, and this successfully limited the inflammation (Lin et al., 2011). However, as mentioned above, 2-APB has been known to interfere with a variety of transport processes besides CRAC channels. In addition, 2-APB is hydrosoluble. Thus, it can enter into the bloodstream and be dispersed via the circulatory system. This may lead to the inhibition of CRAC channels in tissues outside the airway, causing unexpected side effects such as global muscular hypotonia (Vig et al., 2006) (we also observed that after the administration of 2-APB, mice exhibited transient delayed ambulation and slightly reduced muscle strength that lasted for a few seconds to $1 \mathrm{~min}$; unpublished data), chronic pulmonary disease, and congenital myopathy. Further, 2-APB administration only led to a transient remission of AR symptoms.

The use of ORAl1 knock-in mice might better demonstrate the role that SOCE plays in the pathogenesis of AR. However, the absence of ORAl1 in mice can lead to the compromise of mast cell (Vig et al., 2008), B-cell, and T-cell function (Gwack et al., 2008), all of which are indispensable in the process of sensitization. Furthermore, mice that lack ORAl1 have a short postnatal lifespan (with certain variations due to the method and genetic background used for gene targeting).
Therefore, we turned to the method of RNA interference (RNAi), a method often used in gene therapy. RNAi is the docking of specific small RNA molecules with the complementary mRNA. This leads to the formation of a RNA-induced silencing complex that leads to mRNA degradation and the resulting inhibition of translation (Popescu and Popescu, 2007). RNAi could be a more efficient method to block the function of CRAC channels than agents that target Orai1 because multiple copies of Orai1 protein are translated from one mRNA molecule. Lentiviral gene vectors are often used in RNAi in vivo because of their ability to integrate their transgene into host-cell chromosomes, resulting in longer lasting genetic alteration (Cockrell and Kafri, 2007). Respiratory epithelial cells are either slowly dividing or terminally differentiated, rendering them good candidates to achieve prolonged gene silencing, while inflammatory cells are also important targets (Maes et al., 2011). In addition, gene therapy can occur by the direct delivery of gene transfer vectors to the nasal cavity. This allows for a general airway distribution and the potential targeting of different cell types (but mainly epithelial cells) (Griesenbach and Alton, 2009; Gill et al., 2010). We delivered a single dose of a human immunodeficiency virus type I-derived lentiviral vector that contained synthesized anti-ORAl1 shRNA sequences and the GFP gene into the nasal cavity of mice after pre-treatment of the airway with LPC. LPC is a naturally occurring airway surfactant component (Chen et al., 2006), and it can provide the vector deeper access through the transient permeabilization of epithelial tight junctions (Koehler et al., 2005). Minimal in vivo toxicity has been observed for LPC at concentrations that provide strong enhancement of in vivo airway gene transduction into both the surface cells and the deeper cells of the epithelial layer (where the progenitor cell pools reside) (Kremer et al., 2007).

Our observation indicated that a single dose of ORAl1 RNAi lentivirus can achieve a lasting inhibition of Orai1 expression in the nasal mucosa, which could lead to a steady blocking effect on SOCE, and it could reach the peak of the inhibition efficacy three days after the administration of the lentiviruses. The dose of the recombinant lentivirus we chose to administer was decided based on a previous study where we assessed its effect on the expression of Orai1 using Western blotting. In that study, we administered 20 or $40 \mu \mathrm{l}$ of the lentivirus at a titer of $1 \times 10^{8}, 5 \times 10^{8}$, or $1 \times 10^{9} \mathrm{tu} / \mathrm{ml}$ and found that the downregulation of Orai1 was most apparent when a titer of $1 \times 10^{9} \mathrm{tu} / \mathrm{ml}$ was used, with no difference between 20 and $40 \mu \mathrm{l}$ (unpublished 
data). This may be the result of "excess" lentivirus entering into the lower airway. However, the blocking effect might be partial. Only a small percentage of the cells in the epithelial layer were transfected, and the AR-LI mice still had greater Orai1 expression in the nasal mucosa than the normal controls, suggesting that the gene intervention did not lead to the complete absence of CRAC channel function and the resulting complete inability to mount an effective immune response to non-allergens (such as pathogens).

The splenocytes were not found to be transfected, and the Orai 1 mRNA levels in the spleen did not significantly change after lenti-ORAl1 treatment. Therefore, it is reasonable to deduce that the locally delivered lenti-ORA/1 probably did not (and cannot) enter into the bloodstream and did not affect SOCE in other systems. If this is true, it would practically guarantee that the use of lenti-ORAl1 would have a much lower risk of causing side effects than 2-APB. In agreement with this, we did not observe any delayed ambulation or reduced muscle strength after the administration of lenti-ORAI1. However, we did find that Orai1 expression was still slightly decreased in the spleen. This finding cannot be explained based on the other findings of this study, but it can be hypothesized that this alteration was secondary to the reduction of Orai1 expression in the nasal mucosa.

The allergic symptoms were alleviated after the local interference of Orai1 expression, and the lymphocytes, neutrophils, eosinophils and epithelial cells in the NLF were also reduced. This may be due to a decrease of the intensity of the responses by the transfected epithelial cells. The cells that reside in the epithelial layer of the nasal mucosa of mice with AR include nasal epithelial cells (NECs), and some infiltrating dendritic cells, mast cells, T cells, B cells, and eosinophils. SOCE and I CRAC have been observed in all of these cell types except eosinophils (Hoth and Penner, 1992; Zweifach and Lewis, 1993; Hsu et al., 2001; Liu et al., 2005). In the present study, we found that the lenti-ORAl1 can only transfected the cells in the anterior regions of the epithelial layer of the nasal mucosa. The timing of transfection with lenti-ORAl1 we choose was three days before the first challenge. Using the immunofluorescence method, we didn't observe the infiltration of T cells, B cells, mast cells and eosinophils in the epithelial layer of the nasal mucosa when the mice received three times of sensitization but were not challenged yet. Therefore, most of the transfected cells were NECs, instead of the inflammatory cells we hypothesized before.

In NECs, SOCE is the predominant cause of $\mathrm{Ca}^{2+}$ influx. It can regulate secretion by NECs, both the discharge of proteins and the regulation of the transepithelial secretion of salts and water (Parekh and Putney, 2005). NECs from patients with AR could amplify the allergic reaction and contribute to ongoing allergic inflammation via their capacity to act as APCs through the increased expression of HLA-DR and CD86 (Takizawa et al., 2007). They can also attract and activate DCs for the initiation of Th2 immune responses (Hammad and Lambrecht, 2008) and help shape Th2 responses by directly attracting and activating Th2 cells, natural killer T cells, eosinophils, basophils, and mast cells. NECs are in direct contact with the inhalant allergens; due to the presence of memory $T$ cells in the epithelial layer and the capacity of NECs to induce the antigen-specific proliferation of $T$ cells, NECs may play an even more important role in allergic inflammation than what has been demonstrated (Takizawa et al., 2007). The cytokines IL-17E, IL-33, and TSLP lie upstream of the classical Th2 cytokines, and the fact that they are all produced by ECs provides evidence at the molecular level for how ECs can directly impact Th2 immunity (Liu et al., 2007; Saenz et al., 2008). In our study, levels of IL-33 and TSLP expression in the nasal mucosa were both decreased while there was no distinct reduction of IL-17E expression level after the Orai1 expression was downregulated. IL-33 is initially produced by epithelium at the mucosal surfaces in response to challenges, including microflora, allergens, and certain pathogens, and subsequently amplified by other tissue-resident cells and inflammatory cells during inflammatory responses (Bulek et al., 2010). IL-33 has been shown to have a critical function in the initiation and propagation of Th2 immune responses. in vivo, including increased production of Th2 cytokines IL-4, IL-5, IL-13, eosinophilia, and pathological changes in mucosal tissues (Schmitz et al., 2005). Recent studies showed that treatment with anti-IL-33 in model of allergic asthma significantly reduced serum IgE, the numbers of eosinophils and lymphocytes, and concentrations of IL-4, IL-5, and IL-13 in bronchoalveolar lavage fluid (Liu et al., 2009). IL-33 could also increased the survival of eosinophils and induced their superoxide anion production and degranulation. IL-33 induced production of IL-8 and enhanced the viability and adhesiveness of eosinophils (Cherry et al., 2008). Moreover, mast cells are responsive to IL-33. IL-33 was able to stimulate mast cells to produce pro-inflammatory mediators, cytokines, and chemokines including IL-4, IL-13, IL-1b, GM-CSF, TNF-a, MCP-1, IL-8, and IL-6 (Moulin et al., 2007). IL-33 enhanced the maturation and survival of mast cells (Allakhverdi et al., 2007). EC-derived TSLP directly 
activates DCs to generate Th2 permissive microenvironment. TSLP induces the expression of chemokines thymus and activation regulated chemokine and macrophage-derived chemokine in CD11 $\mathrm{C}^{+} \mathrm{DC}$ subset. Those chemokines are ligands for CCR4, which is predominantly found on Th2-type lymphocytes. Furthermore, TSLP also induced the expression of OX40 ligand on DCs, which is required for inflammatory Th2 differentiation. TSLP-activated DCs direct naive Th cells to produce the pro-inflammatory cytokines including IL-4, IL-5, IL-13, and TNFa, but not IL-10 and IFN $\gamma$. Mast cells also express TSLPR and respond to TSLP by producing high levels of Th2 cytokines (Soumelis et al., 2002; Rimoldi et al., 2005).

Therefore, although the lenti-Orai1 could only transfected and hamper the transcripiton of Orai1 in NECs, through inhibiting the secrection of IL-33 and TSLP by NECs, it could have resulted in lessened Th2, mast cells and eosinophils responses and also reduced Orai1 expression in these cells.

T-cell motility during TCR-mediated antigen recognition and formation of the immunological synapse is regulated by SOCE (Delon et al., 1998; Bhakta et al., 2005), which plays an important role in the differentiation of naive $\mathrm{CD} 4{ }^{+} \mathrm{T}$ cells into Th2 cells (Leitenberg and Bottomly, 1999). A sustained increase in intracellular $\mathrm{Ca}^{2+}$ in the presence of costimulatory signals is necessary for a $T$ cell to become activated and not unresponsive to future TCR stimulation (Borde et al., 2006; Bandyopadhyay et al., 2007). The expression of IL-4 was decreased in Th2 cells of Orai1-deficient mice (Gwack et al., 2008). IL-4 can activate the transcription of germline genes and accelerate the class switch recombination to $\operatorname{lgE}$ in local tissues (Fear et al., 2004) through promoting the expression of cytidine deaminase, a protein normally expressed only in B cells in the germinal centers of lymphoid tissues (Muramatsu et al., 2000). Thus, when the IL-4 level is decreased, class switch recombination in local tissues is lessened and ovalbumin-specific IgE production may be reduced. In fact, this is in agreement with our findings in the nasal mucosa, serum, and the germinal centers of spleen. Therefore, it is logical to suggest that lessened SOCE may inhibit Th2 responses not only by inhibiting the activation of NECs but also by hampering the activation and proliferation of Th2 cells. The alteration of eosinophil function that we observed might be subsequent to an impaired NECs and Th2 cells response. Interestingly, the ECP level in the NLF did not significantly change after treatment, even though its mRNA level in the nasal mucosa was reduced. This discrepancy needs further study, but it probably due to the ECP protein having a longer half-life than its mRNA.

In the absence of Orai1, B cells exhibited poor proliferation in response to BCR activation, suggesting that $\mathrm{Ca}^{2+}$ signals are essential for antigen-dependent expansion of $B$ cells in mice (Gwack et al., 2008). While immunoglobulin levels were normal, Orai1-deficient patients failed to mount antigen-specific antibody responses. These past findings help explain our results that showed reduced levels of local and systemic OVA-IgE after lenti-ORAI1 administration and give further evidence why the inhibition of Orai1 hindered allergic pathogenesis.

From the indirect effects that stem from the inhibition of NEC secretion and Th2 responses, reduced Orai1 expression in the airway can also inhibit mast cell hypperreactivity. In mast cells, $\mathrm{Ca}^{2+}$ signals were shown to be involved in FceRI-mediated degranulation and the release of histamine, leukotrienes, and prostaglandins (Baba et al., 2008; Vig et al., 2008), all of which in vitro are impaired in Orai1 $^{-/-}$mice (Vig et al., 2008). Therefore, impaired expression of Orai1 in mast cells in the nasal mucosa may partially account for the decrease in LTC4 production.

In AR-LI mice, it was demonstrated that the local inhibition of Orai1 expression had effects on systemic allergic responses, as there were reductions in inflammatory proteins in the serum and their corresponding mRNA transcripts in the spleen. This may be related to the circulation of the immune cells mentioned above, and suggests that local administration of lenti-ORA/1 may partially block the immunopathological cascade of allergic reactions.

In summary, the present study was a preliminary in vivo investigation on how the downregulation of Orai1 expression in the airway by the administration of a siRNA-containing lentiviral vector would affect the local and systemic levels of several allergy-related cytokines and OVA-lgE. The results suggested that inhibiting the function of CRAC channels in the airway might be a novel and effective therapy for AR. However, whether this treatment can correct the Th1/Th2 imbalance in allergic disease for an extended time period and can work as a prophylactic measure are questions that remain to be answered. Further, it is not known if this intranasal transgenic therapy has the potential to completely eradicate the ability to mount an immune response to pathogens in the airway. Further research should be done to answer these questions in the future to develop more targeted and lower risk therapeutic agents for AR. 


\section{Methods}

\section{shRNA template design and preparation}

The interfering sequences that corresponded to the ORAl1 gene were chosen from the mouse orai1 mRNA sequence (GenBank Accession No. NM_175423) with the use of the siRNA target finder and design tool available at http://www.ambion.com. BLASTN searches were conducted on the sequence to ensure gene specificity. The sequences were designed to include a Mlul restriction site, a 21 nucleotide (nt) sense template (5'-TGAATCAGTCAT CGTCGCCAA-3'), a 9-nt loop (5'-TTCAAGAGA-3'), a $21 \mathrm{nt}$ antisense template (5'-TTGGCGACGATGACTGATTCA$\left.3^{\prime}\right)$, a terminator sequence, and a Clal restriction site. The sequences were chemically synthesized by Sangon (Shanghai, China).

\section{Lentivirus production and determination of viral transfection efficacy and inhibition efficacy of Orai1 transcription}

The expression lentiviral vector pLVTHM (Tronolab, Lausanne, Switzerland) that carried the GFP reporter gene was restriction digested by the restriction enzymes Mlul and Clal (Takara, Japan) and gel purified. The synthesized shRNA sequences were also digested by the two enzymes and then inserted into the Mlul-Clal sites of the vector by T4 DNA ligase (Toyobo, Japan). The resultant clones were verified by sequencing.

We cultured 293T cells (ATCC, Manassas, VA) in Dulbecco's modified Eagle medium supplemented with $10 \%$ fetal bovine serum (Invitrogen, Carlsbad, CA) in 5\% $\mathrm{CO}_{2}$ at $37^{\circ} \mathrm{C}$. The recombinant lentiviruses were produced by the transient transfection of 293T cells. The 293T cells were seeded on $15-\mathrm{cm}$ Petri dishes and were cotransfected at $60-70 \%$ confluence with $20 \mu \mathrm{g}$ of the interferential plasmid, $10 \mu \mathrm{g}$ of the pRsv-REV plasmid, $15 \mu \mathrm{g}$ of the pMDLg-pRRE plasmid, and $7.5 \mu \mathrm{g}$ of the pMD2.G plasmid (Tronolab) according to standard protocols. Transfections were carried out with Lipofectamine 2000 (Invitrogen) as recommended by the manufacturer. The medium containing the recombinant lentivirus was changed once at $12 \mathrm{~h}$ and harvested at $72 \mathrm{~h}$. This collected media was centrifuged at $4000 \mathrm{~g}$ at $4^{\circ} \mathrm{C}$ for $10 \mathrm{~min}$, the resulting supernatant was centrifuged at $72000 \mathrm{~g}$ at $4^{\circ} \mathrm{C}$ for $2 \mathrm{~h}$, and the pellet was then resuspended in phosphate-buffered saline (PBS). Viral stocks were stored at $-80^{\circ} \mathrm{C}$.

To determine the viral transfection efficacy, PANC cells were plated on $6 \mathrm{~cm}$ dishes and infected with lenti-Orai1 and lenti-GFP, which are lentiviral vectors without shRNA, respctively, and another $8 \mu \mathrm{g} / \mathrm{ml}$ of polybrene (Sigma-Aldrich, St. Louis, MO). Forty-eight $h$ after the transduction, the cells were harvested, washed, and analyzed by FACS for GFP.

To examine the efficacy of the lenti-ORAl1 and lenti-GFP to inhibit the Orai1 transcription, the PANC cells which are untreated, infected with lenti-ORA/1, or infected with lenti-GFP were harvested and their total RNA was extracted with Trizol (Invitrogen). For reverse transcription, 2 $\mu \mathrm{g}$ of the previously measured RNA was reverse transcribed with first strand cDNA synthesis kits (Invitrogen) to complementary DNA according to the manufacturer's instructions. Primers were designed with Primer Express Software (Applied Biosystems, Foster City, CA) based on sequences available in GenBank and were synthesized (Sangon). Real-time RT-PCR was performed to detect the mRNA levels of Orai1. The primers were: forward primer 5'-TTTGCCCTCATGATCAGCAC-3' and reverse primer 5'TTGGCGACGATGACTGATTC-3'. Glyceraldehyde-3-phosphate dehydrogenase (GAPDH) mRNA was examined to control for the sample-to-sample variation in RNA isolation and integrity by using the following pair of primers: forward primer 5'-ACCACAGTCCATGCCATCAC-3' and reverse primer 5'-TCCACCACCCTGTTGCTGTA-3'. Real-time PCR was performed on the ABI Prism 7500 Real-time PCR system (Applied Biosystems) with the SYBR Premix Ex Taq kit (TaKaRa, Japan) in accordance with the manufacturer's instructions. The specificity of the PCR products was evaluated by melting curve analysis. Evaluation of the data was performed by the cycle threshold $\left(2^{-\Delta \Delta \mathrm{CT}}\right)$ method, and GAPDH was used for normalization.

\section{Animals}

Female BALB/c mice (6-week old) were purchased from the Chinese Academy of Sciences Shanghai Laboratory Animal Center. The mice were maintained in horizontal laminar flow cabinets and were provided sterile food and water in a pathogen-free facility. All the animals were handled in accordance with the National Institutes of Health Guidelines for the Care and Use of Laboratory Animals.

\section{In vivo transfection protocols and determination of the kinetics of the inhibition efficacy of Orai1 transcription}

For the lentiviral transfection, the BALB/c mice were anaesthetized with an intramuscular injection of xylazil (12 $\mu \mathrm{g} / \mathrm{g}$ body weight) and ketamine $(40 \mu \mathrm{g} / \mathrm{g})$ prior to the delivery of lysophosphatidylcholine (LPC; in PBS, $0.3 \% \mathrm{w} / \mathrm{v}$; Sigma-Aldrich) to the nasal airway via inhalation-driven instillation $(8 \mu \mathrm{l} / \mathrm{mouse})$. The lentivirus $\left(1 \times 10^{9} \mathrm{tu} / \mathrm{ml}\right)$ were delivered into the nostrils of the mice (two 10- $\mu$ l aliquots were administered to each mouse over a period of several min) $1 \mathrm{~h}$ after LPC delivery (Stocker et al., 2009). Twenty-one mice were randomly choosed, and eighteen of them were transfected with lenti-ORAl1 on Day 0 . Three untransfected mice were sacrificed on day1. The transfected mice were sacrificed on day 1 , day 3 , day 7 , day 14 , day 21 , day 28 , three mice each day. Their nasal mucosas were collected to mesure the mRNA levels of Orai1 in them using real-time RT-PCR.

\section{Grouping, sensitization, challenge, and treatment protocol}

Fifty-nine mice were randomly divided into five groups: normal control $(n=15)$, normal mice with Lenti-Orai1 interferenced (NLI) $(n=7)$, AR $(n=15)$, AR mice with lenti-GFP interferenced (AR-GFP) $(n=7)$, and AR mice with lenti-Orai1 interferenced (AR-LI) $(n=15)$.

Based on a published procedure (Ikeda et al., 2002), 
three of the groups of mice (AR, AR-GFP, and AR-LI) were sensitized to ovalbumin by the administration of $0.5 \mathrm{mg} / \mathrm{ml}$ ovalbumin (Grade V, Sigma-Aldrich) and $20 \mathrm{mg} / \mathrm{ml}$ aluminum hydroxide (Sigma-Aldrich) in saline by intraperitoneal injection at a dosage of $0.2 \mathrm{ml} /$ mouse. The sensitization was repeated weekly for 3 weeks (day 1,8 and 15), followed by the daily delivery of an ovalbumin solution (40 $\mathrm{mg} / \mathrm{ml}$ in saline) into the nostrils via inhalation-driven instillation (20 $\mu \mathrm{l} /$ mouse) on days $22-29$ (the challenge). As controls, the remaining groups of mice received neither sensitization nor challenge, except for one dose of the challenge on day 29 (Figure 1).

On day 19, mice in NLI group, AR-GFP group, AR-LI group received transfection with lenti-ORAl1, lenti-GFP, and lenti-ORA/1 $\left(1 \times 10^{9} \mathrm{tu} / \mathrm{ml}\right)$, respectively.

\section{Examination of the transfected cells by the recombi- nant lentivirus}

To determine if the recombinant lentivirus was successfully transfected into cells in the nasal mucosas of the NLI, AR-GFP and AR-LI mice, three mice in each group were killed on day 22, and their nasal mucosa and spleens were carefully dissected. These tissues were fixed with $4 \%$ buffered paraformaldehyde for $24 \mathrm{~h}$, stained with DAPI and observed under fluorescence microscope to determine if the green fluorescence of GFP was expressed in potentially transfected cells.

\section{Assessment of nasal symptoms, cellular analysis of NLF, and sample preparation}

The nasal symptoms of each mouse were evaluated by counting the number of sneezes and episodes of nasal rubbing during the $10 \mathrm{~min}$ immediately after the last ovalbumin intranasal challenge on day 29. Blood and NLF were collected from each mouse $2 \mathrm{~h}$ after the last challenge. One injection of $1 \mathrm{ml} 0.01 \mathrm{M}$ PBS was delivered into the nasal cavities of each mouse after sacrifice, and the fluid was collected with a tube below both nares (the NLF). The NLF and the coagulated blood were centrifuged, and the supernatants were analyzed by ELISA for levels of LTC4, ECP, OVA-IgE, and IL-4.

The cells in the NLF were resuspended with $1 \mathrm{ml} 0.01 \mathrm{M}$ PBS with $1 \%$ bovine serum albumin (BSA) for cellular analysis. The total cell number of leukocyte in the NLF samples was counted using an hemocytometer (Mindray 3000 , Shenzhen, China). The differential count of cells in NLF was determined by review of Wright-Giemsa stained smear and subclassification of 300 consecutive cells. By Wright-Giemsa staining, the percentages of epithelial cells, lymphocytes, neutrophils and eosinophils were determined.

After the mice were sacrificed, the nasal mucosa and spleens of six mice in Normal, AR and LI group, and of four mice in NLI, AR-GFP group were obtained. These tissues were cut into two portions; one was dissected for isolation, the other for mRNA extraction. After sample preparation, all samples were frozen at $-80^{\circ} \mathrm{C}$.

The heads and spleens of the remaining mice in Normal, AR and LI group were excised and immersed in $4 \%$ buffered paraformaldehyde. After fixation, the heads were decalcified in $10 \%$ ethylenediamine tetraacetic acid for 28 days. The specimens were embedded in paraffin and coronally sectioned at a thickness of $4 \mu \mathrm{m}$.

\section{Western blotting analysis}

The frozen tissues were homogenized, and the proteins in the tissues were extracted with Mouse/Rat Tissue Extract Protein Array Kit (Sigma-Aldrich). Protein homogenates (40 $\mu \mathrm{g} /$ lane) were separated by $12 \%$ SDS-PAGE gel and transferred to polyvinylidene difluoride membrane (Millipore, Billerica, MA). After $1 \mathrm{~h}$ blocking with $5 \%$ non-fat milk, the membrane was incubated with mouse anti-Orai1 monoclonal antibody (Sigma-Aldrich) at $4^{\circ} \mathrm{C}$ overnight. The membrane was incubated with a secondary antibody conjugated to HRP (Jackson ImmnoResearch, West Grove, PA) and developed using chemiluminescence Western blotting system (Sigma-Aldrich). The expressions of IL-33, IL-17E and TSLP were also analyzed using rat anti-mouse IL-33 monoclonal antibody (R\&D systems, Minneapolis, $\mathrm{MN}$ ), rat anti-mouse IL-17E monoclonal antibody (R\&D systems), and rabbit anti-mouse TSLP polyclonal antibody (ProSci Incorporated, Poway, CA), respectively. A mouse anti- $\beta$-actin monoclonal antibody (Sigma-Aldrich) was used to analyze the expression of $\beta$-actin, which was used as an internal control.

\section{Immunohistochemistry}

For immunohistochemical staining, slices were deparaffinized and rehydrated with distilled water. Endogenous peroxidase was blocked with $3 \%$ hydrogen peroxide for 20 min. Normal horse serum (Vector Lab, Burlingame, CA) was used to block non-specific binding, and a previously optimized concentration of mouse anti-Orai1 monoclonal antibody (Sigma-Aldrich) in antibody diluent (Dako, Via Real Carpinteria, CA) was immediately applied to the sections and incubated for $1 \mathrm{~h}$ at room temperature and overnight at $4^{\circ} \mathrm{C}$. Biotinylated anti-mouse IgG secondary antibody (Vector Lab) was applied to each section for $30 \mathrm{~min}$, followed by $20 \mathrm{~min}$ incubation with horseradish peroxidase streptavidin (Vector Lab). Slides were stained with 3,3-diaminobenzidine chromogene (Sigma-Aldrich) for $3 \mathrm{~min}$ and counterstained with hematoxylin. Antibody localization was detected microscopically by brown staining. Immunoreactivity and the cellular distribution of Orai 1 were assessed under light microscopy by two observers who were blinded to the sample groups.

\section{ELISA}

Concentrations of OVA-IgE, LTC4, ECP, and IL-4 in serum and NLF were measured by the corresponding ELISA kits (Blue Gene, Shanghai, China). The ELISAs were performed in accordance with the manufacturer's protocols.

\section{Real-time reverse transcription PCR (real-time RT-PCR)}

Total RNA was extracted from the nasal mucosa and spleen samples with Trizol (Invitrogen). Real-time RT-PCR was performed to detect the mRNA levels of Orai1, LTC4S, 
EAR3, the germline $C \varepsilon$ transcript, and IL-4, using the method mentioned before. The primers were as follows: LTC4S, forward primer 5'-CCTACAGGTGATCTCTGCACG A-3' and reverse primer 5'-TATCCCTGGAAATAGCGGAG G-3'; EAR3, forward primer 5'-TAATGCTTGCCTCATGCCT G-3' and reverse primer 5'-TGCACAAGCCACTTGGATTT-3'; Germline $\mathrm{C} \varepsilon$ transcript, forward primer 5'-TGCATTGAAGG CTACGGCTA-3' and reverse primer 5'-TTGGAGCCATTG GATTTCAG-3'; IL-4, forward primer 5'-ATCGGCATTTTGA ACGAGGT-3' and reverse primer 5'-TCGAAAAGCCCGA AAGAGTC-3'. GAPDH mRNA was examined to control for the sample-to-sample variation in RNA isolation and integrity. Evaluation of the data was performed by the cycle threshold $\left(2^{-\Delta \Delta \mathrm{CT}}\right)$ method, and GAPDH was used for normalization.

\section{Statistical analysis}

We used the unpaired Student's $t$ test \pm Welch's correction to compare sneezes, episodes of nasal rubbing, the expression levels of Orai1, inflammatory cells in the NLF, inflammatory cytokine levels, and OVA-IgE levels between the three groups (normal, AR, and LI). Statistical analysis was performed using commercially available statistical software SPSS 17.0 (SPSS, Chicago, IL). Data are presented as mean \pm standard error of the mean (SEM). P values of $<0.05$ were considered to be statistically significant.

\section{Acknowledgements}

This work was supported by the National Natural Science Foundation of China (Grant No. 30973281).

\section{References}

Allakhverdi Z, Smith DE, Comeau MR, Delespesse G. Cutting edge: the ST2 ligand IL-33 potently activates and drives maturation of human mast cells. J Immunol 2007; 179:2051-4

Baba Y, Nishida K, Fujii Y, Hirano T, Hikida M, Kurosaki T. Essential function for the calcium sensor STIM1 in mast cell activation and anaphylactic responses. Nat Immunol 2008; 9:81-8

Bandyopadhyay S, Soto-Nieves N, Macián F. Transcriptional regulation of T cell tolerance. Semin Immunol 2007;19:180-7

Bhakta NR, Oh DY, Lewis RS. Calcium oscillations regulate thymocyte motility during positive selection in the three-dimensional thymic environment. Nat Immunol 2005;6:143-51

Borde M, Barrington RA, Heissmeyer V, Carroll MC, Rao A. Transcriptional basis of lymphocyte tolerance. Immunol Rev 2006;210:105-19

Bulek K, Swaidani S, Aronica M, Li XX. Epithelium: the interplay between innate and Th2 immunity. Immunol Cell Biol 2010;88:257-68

Chen X, Hyatt BA, Mucenski ML, Mason RJ, Shannon JM. Identification and characterization of a lysophosphatidylcho- line acyltransferase in alveolar type II cells. Proc Natl Acad Sci USA 2006;103:11724-9

Cherry WB, Yoon J, Bartemes KR, lijima K, Kita H. A novel IL-1 family cytokine, IL-33, potently activates human eosinophils. J Allergy Clin Immunol 2008;121:1484-90

Cockrell AS, Kafri T. Gene delivery by lentivirus vectors. Mol Biotechnol 2007;36:184-204

Delon J, Bercovici N, Liblau R, Trautmann A. Imaging antigen recognition by naive CD4+ T cells: compulsory cytoskeletal alterations for the triggering of an intracellular calcium response. Eur J Immunol 1998;28:716-29

Fear DJ, McCloskey N, O'Connor B, Felsenfeld G, Gould HJ. Transcription of Ig germline genes in single human $B$ cells and the role of cytokines in isotype determination. $\mathrm{J} \mathrm{Immunol}$ 2004;173:4529-38

Feske S. Calcium signalling in lymphocyte activation and disease. Nat Rev Immunol 2007;7:690-702

Feske S. ORAI1 and STIM1 deficiency in human and mice: roles of store-operated $\mathrm{Ca}^{2+}$ entry in the immune system and beyond. Immunol Rev 2009;231:189-209

Gill DR, Bazzani RP, Hyde SC. Strategies for long-term expression of transgenes in the respiratory epithelium. Curr Opin Mol Ther 2010;12:386-93

Griesenbach U, Alton EW. Gene transfer to the lung: lessons learned frommore than 2 decades of CF gene therapy. Adv Drug Deliv Rev 2009;61:128-39

Gwack Y, Feske S, Srikanth S, Hogan PG, Rao A. Signaling to transcription: store-operated $\mathrm{Ca}^{2+}$ entry and NFAT activation in lymphocytes. Cell Calcium 2007;42:145-56

Gwack Y, Srikanth S, Oh-Hora M, Hogan PG, Lamperti ED, Yamashita M, Gelinas C, Neems DS, Sasaki Y, Feske S, Prakriya M, Rajewsky K, Rao A. Hair loss and defective Tand B-cell function in mice lacking ORAl1. Mol Cell Biol 2008;28:5209-22

Hammad H, Lambrecht BN. Dendritic cells and epithelial cells: linking innate and adaptive immunity in asthma. Nat Rev Immunol 2008;8:193-204

Hoth M, Penner R. Depletion of intracellular calcium stores activates a calcium current in mast cells. Nature 1992; 355:353-6

Hsu Sf, O'Connell PJ, Klyachko VA, Badminton MN, Thomson AW, Jackson MB, Clapham DE, Ahern GP. Fundamental $\mathrm{Ca}^{2+}$ signaling mechanisms in mouse dendritic cells: CRAC is the major $\mathrm{Ca}^{2+}$ entry pathway. $\mathrm{J}$ Immunol 2001;166:6126-33

Ikeda Y, Kaneko A, Yamamoto M, Ishige A, Sasaki H. Possible involvement of suppression of Th2 differentiation in the anti-allergic effect of Shoseiryuto in mice. Jpn J Pharmacol 2002; $90: 328-36$

Koehler DR, Frndova H, Leung K, Louca E, Palmer D, Ng P, McKerlie C, Cox P, Coates AL, Hu J. Aerosol delivery of an enhanced helper-dependent adenovirus formulation to rabbit lung using an intratracheal catheter. J Gene Med 2005; $7: 1409-20$

Kremer KL, Dunning KR, Parsons DW, Anson DS. Gene delivery to airway epithelial cells in vivo: a direct comparison of 
apical and basolateral transduction strategies using pseudotyped lentivirus vectors. J Gene Med 2007;9:362-8

Leitenberg D, Bottomly K. Regulation of naive T cell differentiation by varying the potency of TCR signal transduction. Semin Immunol 1999;11:283-92

Lin L, Zheng C, Zhang L, Da C, Zhao K. Up-regulation of Orai1 in murine allergic rhinitis. Histochem Cell Biol 2010;134 93-102

Lin L, Zheng C, Zhang L, Da C, Zhao K. 2-Aminoethoxydiphenyl borate administration into the nostril alleviates murine allergic rhinitis. Am J Otolaryngol 2011;32:318-28

Liou J, Kim ML, Heo WD, Jones JT, Myers JW, Ferrell JE, et al. STIM is a $\mathrm{Ca}^{2+}$ sensor essential for $\mathrm{Ca}^{2+}$-store-depletion-triggered $\mathrm{Ca}^{2+}$ influx. Curr Biol 2005;15:1235-41

Liu QH, Liu X, Wen Z, Hondowicz B, King L, Monroe J, Freedman $\mathrm{BD}$. Distinct calcium channels regulate responses of primary $B$ lymphocytes to $B$ cell receptor engagement and mechanical stimuli. J Immunol 2005;174:68-79

Liu X, Li M, Wu Y, Zhou Y, Zeng L, Huang T. Anti-IL-33 antibody treatment inhibits airway inflammation in a murine model of allergic asthma. Biochem Biophys Res Commun 2009; $386: 181-5$

Liu YJ, Soumelis V, Watanabe N, Ito T, Wang YH, Malefyt RW, Omori M, Zhou BH, Ziegler SF. TSLP: an epithelial cell cytokine that regulates $T$ cell differentiation by conditioning dendritic cell maturation. Annu Rev Immunol 2007;25:193-219

Maes T, Tournoy KG, Joos GF. Gene therapy for allergic airway diseases. Curr Allergy Asthma Rep 2011;11:163-72

Mercer JC, Dehaven WI, Smyth JT, Wedel B, Boyles RR, Bird GS, Putney JW Jr. Large store-operated calcium-selective currents due to co-expression of Orai1 or Orai2 with the intracellular calcium sensor, Stim1. J Biol Chem 2006;281:24979-90

Moulin D, Donze O, Talabot-Ayer D, Mezin F, Palmer G, Gabay C. Interleukin (IL)-33 induces the release of pro-inflammatory mediators by mast cells. Cytokines 2007;40:216-25

Muramatsu M, Kinoshita K, Fagarasan S, Yamada S, Shinkai $\mathrm{Y}$, Honjo T. Class switch recombination and hypermutation require activation-induced cytidine deaminase (AID), a potential RNA editing enzyme. Cell 2000;102:553-63

Parekh AB, Putney JW. Store-operated calcium channels. Physiol Rev 2005;85:757-810

Popescu FD, Popescu F. A review of antisense therapeutic interventions for molecular biological targets in asthma. Biologics 2007;1:271-83

Prakriya M, Lewis RS. Potentiation and inhibition of $\mathrm{Ca}^{2+}$ release-activated $\mathrm{Ca}^{2+}$ channels by 2-aminoethyldiphenyl borate (2-APB) occurs independently of IP3 receptors. J Physiol 2001;536:3-19
Prakriya M, Feske S, Gwack Y, Srikanth S, Rao A, Hogan PG. Orai1 is an essential pore subunit of the CRAC channel. Nature 2006;443:230-3

Rimoldi M, Chieppa M, Salucci V, Avogadri F, Sonzogni A, Sampietro GM, Nespoli A, Viale G, Allavena P, Rescigno M. Intestinal immune homeostasis is regulated by the crosstalk between epithelial cells and dendritic cells. Nat Immunol 2005;6:507-14

Saenz SA, Taylor BC, Artis D. Welcome to the neighborhood: epithelial cell-derived cytokines license innate and adaptive immune responses at mucosal sites. Immunol Rev 2008; 226:172-90

Schmitz J, Owyang A, Oldham E, Song Y, Murphy E, McClanahan TK, Zurawski G, Moshrefi M, Qin JZ, Li XX Gorman DM, Bazan JF, Kastelein RA. IL-33, an interleukin-1-like cytokine that signals via the IL-1 receptor-related protein ST2 and induces T helper type 2-associated cytokines. Immunity 2005;23:479-90

Soumelis V, Reche PA, Kanzler H, Yuan W, Edward G, Homey B, Gilliet M, Ho S, Antonenko S, Lauerma A, Smith K, Gorman D, Zurawski S, Abrams J, Menon S, McClanahan T, de Waal-Malefyt R, Bazan F, Kastelein RA, Liu YJ. Human epithelial cells trigger dendritic cell mediated allergic inflammation by producing TSLP. Nat Immunol 2002;3:673-80

Stocker AG, Kremer KL, Koldej R, Miller DS, Anson DS, Parsons DW. Single-dose lentiviral gene transfer for lifetime airway gene expression. J Gene Med 2009;11:861-7

Takizawa R, Pawankar R, Yamagishi S, Takenaka H, Yagi T. Increased expression of HLA-DR and CD86 in nasal epithelial cells in allergic rhinitics: antigen presentation to T cells and up-regulation by diesel exhaust particles. Clin Exp Allergy 2007;37:420-33

Vig $M$, Peinelt C, Beck A, Koomoa DL, Rabah D, Koblan-Huberson M, Kraft S, Turner H, Fleig A, Penner R, Kinet JP. CRACM1 is a plasma membrane protein essential for store-operated $\mathrm{Ca}^{2+}$ entry. Science 2006;312:1220-3

Vig M, DeHaven WI, Bird GS, Billingsley JM, Wang H, Rao PE, Hutchings AB, Jouvin M, Putney JW, Kinet JP. Defective mast cell effector functions in mice lacking the CRACM1 pore subunit of store-operated calcium release-activated calcium channels. Nat Immunol 2008;9:89-96

Wang Y, Deshpande M, Payne R. 2-Aminoethoxydiphenyl borate inhibits phototransduction and blocks voltage-gated potassium channels in Limulus ventral photoreceptors. Cell Calcium 2002;32:209-16

Zweifach A, Lewis RS. Mitogen-regulated $\mathrm{Ca}^{2+}$ current of T lymphocytes is activated by depletion of intracellular $\mathrm{Ca}^{2+}$ stores. Proc Natl Acad Sci USA 1993;90:6295-9 